\title{
A pathway of signals regulating effector and initiator caspases in the developing Drosophila eye
}

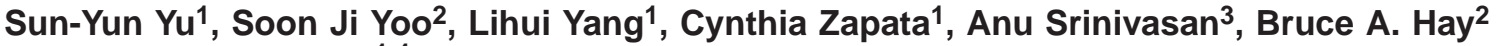 \\ and Nicholas E. Baker 1,4 \\ ${ }^{1}$ Department of Molecular Genetics, Albert Einstein College of Medicine, 1300 Morris Park Avenue, Bronx, NY 10461, USA \\ 2Division of Biology, California Institute of Technology, 1200 East California Boulevard, Pasadena, CA 91125, USA \\ 3IDUN Pharmaceuticals, 11085 N. Torrey Pines Road, Suite 300, La Jolla, CA 92037, USA \\ *Author for correspondence (e-mail: baker@aecom.yu.edu)
}

Accepted 22 April 2002

\section{SUMMARY}

Regulated cell death and survival play important roles in neural development. Extracellular signals are presumed to regulate seven apparent caspases to determine the final structure of the nervous system. In the eye, the EGF receptor, Notch, and intact primary pigment and cone cells have been implicated in survival or death signals. An antibody raised against a peptide from human caspase 3 was used to investigate how extracellular signals controlled spatial patterning of cell death. The antibody crossreacted specifically with dying Drosophila cells and labelled the activated effector caspase Drice. It was found that the initiator caspase Dronc and the proapoptotic gene head involution defective were important for activation in vivo. Dronc may play roles in dying cells in addition to activating downstream effector caspases. Epistasis experiments ordered EGF receptor, Notch, and primary pigment and cone cells into a single pathway that affected caspase activity in pupal retina through hid and Inhibitor of Apoptosis Proteins. None of these extracellular signals appeared to act by initiating caspase activation independently of hid. Taken together, these findings indicate that in eye development spatial regulation of cell death and survival is integrated through a single intracellular pathway.

Key words: Drosophila eye, Caspase, Drice, Dronc, Notch, EGF receptor, Hid, IAP, Apoptosis

\section{INTRODUCTION}

During development, programmed cell death is regulated by cell-cell signals that either promote or inhibit caspase activation (Meier et al., 2000a). The biochemical mechanisms of extracellular regulation are incompletely known. Here, they have been studied using an antibody to detect caspase activation in vivo. Caspases are a class of intracellular cysteine proteases that cause cell death by cleaving a variety of intracellular proteins after aspartate residues (Chinnaiyan and Dixit, 1996). Accordingly, inhibitors of the caspase enzymes can prevent cell death (Bump et al., 1995; Xue and Horvitz, 1995; Ekert et al., 1999). Extracellular signals can affect caspase activity either through the activation of caspase zymogens, or through inhibition of caspase enzymatic activity.

Cleavage of caspase zymogen permits assembly of active caspase heterodimer from proteolytically generated p10 and p20 subunits. Caspases are broadly divided into two classes. Initiator caspases do not require other caspases for cleavage of their zymogens, but are thought to interact with other regulators through their prodomains. Initiator caspases may be activated by cell surface receptors, cytochrome c release from mitochondria, the balance of positively and negatively acting
Bcl family members, and ced-4/Apaf like proteins. Effector caspases are activated by other caspases, lack extensive prodomains and are thought to be responsible for the bulk of cellular proteolysis. It is thought that effector caspases may cleave their own zymogens to provide positive feedback once apoptosis has been initiated (Cryns and Yuan, 1998; Kumar, 1999; Budihardjo et al., 1999; Salvesen and Dixit, 1999; Song and Steller, 1999).

In Drosophila, proapoptotic genes have been found that are required for most programmed cell death in the embryo. Three genes reaper (rpr), grim and head involution defective (hid) map close together on chromosome 3 (bands $75 \mathrm{C} 1,2$ ), and all are deleted by a small deficiency called H99. There is no programmed cell death in H99 homozygous embryos (White et al., 1994; Song and Steller, 1999). The rpr and grim genes are transcribed exclusively in cells fated to die, and their promoters must respond to extracellular signals controlling apoptosis (Nordstrom et al., 1996). The hid gene is also expressed in cells that survive, and could be regulated through MAP kinase by extracellular signals and receptor tyrosine kinases (Bergmann et al., 1998; Kurada and White, 1998). rpr, grim and hid encode intracellular proteins that promote apoptosis by inhibiting Inhibitor of Apoptosis Proteins (IAPs). 
DIAP1 (Th - FlyBase) and DIAP2 (Iap2 - FlyBase) are two Drosophila IAPs that prevent cell death on overexpression (Hay et al., 1995). DIAP1 binds to activated caspases and to inactive zymogens, and has been found able to inhibit both caspase activation and caspase activity. Inhibition of caspase activation is more important in vivo (Hawkins et al., 1999; Wang et al., 1999; LaCount et al., 2000). Biochemical experiments and genetic epistasis both indicate that $\mathrm{rpr}$, grim and hid prevent DIAP1 from inhibiting caspases, and suggest that this is the mechanism by which cell death is promoted, not through initiating caspase activation (Wang et al., 1999; Goyal et al., 2000; Meier et al., 2000b).

Extracellular signals regulate apoptosis during Drosophila eye development (Rusconi et al., 2000; Baker, 2001). After the morphogenetic furrow passes across the eye disc, ommatidial signals activate the EGF receptor to maintain survival (Baker and Yu, 2001). After most ommatidial cell types have been specified, cell death later removes unwanted cells during pupation. On average two to three cells are lost from the cells that surround each ommatidium. Interommatidial cells that survive this death episode go on to differentiate as the pigment cells and sensory bristle cell types that separate the ommatidia in the adult eye (Wolff and Ready, 1991; Hay et al., 1994). A signal from differentiating cone cells or primary pigment cells as well as activity of the EGF receptor appear to confer interommatidial cell survival in the pupa (Sawamoto et al., 1998; Miller and Cagan, 1998). The cone or primary pigment cell signal inhibits $\operatorname{Notch}(N)$ activity (Miller and Cagan, 1998). $N$ is required for cell death and therefore might act to initiate caspase activation (Cagan and Ready, 1989; Wolff and Ready, 1991; Rusconi et al., 2000). hid is also required for cell death (Kurada and White, 1998).

Recently, a polyclonal antiserum specific for an activated caspase was obtained by immunising rabbits with a 13-amino acid peptide corresponding to the $\mathrm{C}$ terminus of the large subunit of human and mouse caspase 3 . The affinity-purified CM1 antiserum recognises the large subunit of processed caspase 3 but not unprocessed zymogen on western blots, and specifically immunostains apoptotic neurones in vitro and in vivo (Srinivasan et al., 1998). The CM1 antibody has been widely used for in situ detection of caspase activation during physiological and pathological apoptosis in mammalian cells (Hartmann et al., 2000; Finn et al., 2000; Chong et al., 2000; Jones et al., 2000; Bialik et al., 1999; Gu et al., 1999; Krebs et al., 1999). The CM1 antibody has been used in Drosophila to investigate the autonomy of EGFR signalling in survival (Baker and Yu, 2001). We report that the CM1 antiserum crossreacted with activated Drice (Ice - FlyBase) and specifically labelled apoptotic cells in Drosophila. We used this reagent to probe cell death in the pupal retina and also in the larval eye imaginal disc. In normal eye development, all cell death depended on activation of effector caspases by the initiator caspase Dronc ( $\mathrm{Nc}-$ FlyBase) along with at least one p35-sensitive initiator caspase. Dronc may also cleave other targets in vivo in addition to effector caspases. We found that hid function was absolutely required for programmed cell death in the developing eye, and that hid and DIAP1 affect caspase activation, in contrast to the caspase inhibitor baculovirus $\mathrm{p} 35$. The EGFR promoted survival by preventing initiator caspase function. Such survival signalling acted entirely by inactivating hid. The proapoptotic role of $N$ was found to block EGFR-dependent survival signalling and was dispensable in the absence of EGFR. The cone cell- or primary pigment cell-derived survival signal that inhibits $N$ must act upstream of the role of $N$ in blocking EGF receptor. Thus, regulators of cell death acted through a single pathway that affected caspase activity through Hid; none were found to promote initiator caspase function more directly.

\section{MATERIALS AND METHODS}

\section{Drosophila strains}

The following Drosophila strains have been described previously:

GMRp35 (Hay et al., 1994); GMRDIAP1 (Hay et al., 1995); GMRDronc and GMRDronc-DN (Hawkins et al., 2000) (each encodes a zymogen that requires activation); Df (3L)H99 (White et al., 1994); $N^{l 1-N t s l}$ and $N^{f a-g}$ (Cagan and Ready, 1989); Ellipse $\left(\right.$ egfrE1 legfr ${ }^{E 3}$ ) (Baker and Rubin, 1992); and egfrf ${ }^{24}$ (also known as top $^{C O}$ ) and egfrtsla (Kumar et al., 1998). The hid mutant genotype was $l(3) k 05017 / \mathrm{Df}(3 \mathrm{~L}) W^{R+X 1}$ (Grether et al., 1995).

\section{Antibody procedures}

Antibody labelling was performed according to standard procedures. CM1 antiserum was used diluted 1:1000 in $0.1 \mathrm{M}$ sodium phosphate (pH7.2), 5\% normal goat serum, 0.1\% saponin. Embryos and pupal retinas were fixed (20 minutes, room temperature) in $4 \%$ formaldehyde in $0.1 \mathrm{M}$ sodium phosphate ( $\mathrm{pH}$ 7.2). Embryos were fixed in the presence of n-heptane. Eye imaginal discs were fixed in PLP (Tomlinson and Ready, 1987). Confocal microscopy was performed using BioRad MRC600 and Radiance 2000 instruments.

pET23a-Dcp1 (full length), pET23a-Dronc (full length) and pet23a-DriceD217E, each of which encodes a C-terminal His6 tag, were expressed individually in BL21/pLysS. Each caspase was affinity purified using Ni-Agarose (Qiagen) as described (Hawkins et al., 2000). The caspases were dialysed into $20 \mathrm{mM}$ Tris (pH 7.5), 100 $\mathrm{mM} \mathrm{NaCl}, 1 \mathrm{mM}$ DTT, $10 \%$ glycerol after elution with $20 \mathrm{mM}$ imidazole. Caspases (100 ng) were loaded onto $15 \%$ gels and transferred to nitrocellulose for western blotting. CM1 was diluted 1:2000, incubated with blots for 1 hour as described previously (Srinivasan et al., 1998) and visualised by ECL (Pierce). Fivefold more of the caspases was used for Coomassie staining.

\section{RESULTS}

\section{Labelling dying cells in Drosophila with an antibody against a human caspase}

A polyclonal rabbit antiserum (CM1) has been raised to a 13 amino acid peptide corresponding to the $\mathrm{C}$ terminus of human caspase 3 (Srinivasan et al., 1998). Drosophila embryos were stained with CM1 antibody to test whether caspase activation could be detected in situ. From stage 11 onwards, an evolving pattern of labelled cells was seen in the head, trunk epidermis and CNS (Fig. 1A,B). CM1 labelling was completely dependent on the chromosomal region $75 \mathrm{C} 1,2$, as embryos homozygous for $D f(3 \mathrm{~L}) \mathrm{H} 99$ were unstained (data not shown). H99 homozygous embryos lack apoptosis because of the deletion of the reaper, grim and hid genes. Thus, the CM1 antibody staining corresponded to dying cells.

Fig. 1D shows an alignment of the $\mathrm{C}$ terminus of the large subunit of human and mouse caspase 3, to Spodoptera frugiperda caspase 1 and the corresponding regions of Drosophila caspase genes. Most similar is the effector caspase 

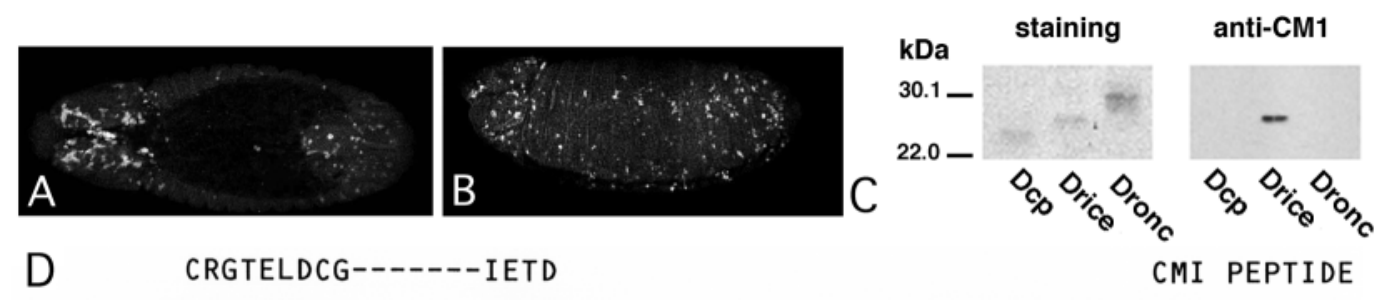

KPKLFFIQACQGDRLDGGITLSR--TETDGSP---------STSYRIPVHADFL SFCASPASE-1

KPKLFFIQACQGDRLDGGVTMQRSQTETDGDS----------SMSYKIPVHADFL DRICE KPKLFF IQACQCDRLDGGITLEKGVTETDGESKPKLFFIQACRGANLEKAVEFSSFAVMTRELVPEP-----AAAVQPI TYA IPSTADIL DECAY KPKILIVQACKGPLRADAKKMNNEGYLSYRNENHGSVFIQLCDVMDQYGLTRDFQS I FKH DAMM KPKLLI IQACQEKLVHKKKPNEL FRIDVTTVS-PPKLI FVQACKGDCQLGGFMTDAAQPNGSPNEILKCYSTYEGFVSFRTEDGLNRSGKTSD DREAM KPKVLMFPFCRGDEYDLGHPKNQGNLMEPVYTAQEEKWPDTQTEGIPSPSTNVPSLADTL DRONC

Fig. 1. Embryos labelled with CM1 antiserum. Anterior towards the left in these and subsequent figures. (A) Wild type, stage 12. (B) Wild type, stage 15. (C) In western blots, CM1 recognised cleaved Drice but not cleaved Dronc or DCP1. (D) Alignment of the putative p20/p10 linker regions from Drosophila caspases with the human caspase 3 peptide and with caspase 1 from $S$. frugiperda, for which cleavage has been demonstrated C-terminal to either aspartate residue (Ahmad et al., 1997). Residues identical to the 13-mer CM1 peptide shaded (gaps has been introduced to permit alignment of active sites and p20 C termini). Long pro-regions suggest Decay, Damm, Dredd, Dream and Dronc are likely to be regulated initiator caspases.

Drice, with eight out of 13 identical residues. The next most similar are DCP-1 (seven out of 13) and Dronc (six out of 13). Western blotting experiments were performed to test whether CM1 antiserum might cross react with activated caspases from Drosophila. CM1 recognised activated Drice but not activated DCP-1, Dronc or any of the three inactive zymogens (Fig. 1C).

\section{Caspase antibody labelling of the pupal retina}

CM1 staining precisely mirrored the pattern of cell death described previously for the pupal retina (Wolff and Ready, 1991). A burst of CM1 immunoreactivity in cells surrounding ommatidial clusters peaked at 30 hours after puparium formation (APF; pupae maintained at $25^{\circ} \mathrm{C}$; Fig. 2A). This was replaced by CM1 staining of the retinal margin between 40 and 48 hours APF (at $25^{\circ} \mathrm{C}$ ) (Fig. 2B).

Since retinas homozygous for $D f$ (3L)H99 cannot be obtained, expression of the baculovirus protein p35 was used to test whether CM1 staining reflected caspase activation in dying retinal cells. Despite the lack of cell death in GMRp35 retina (Hay et al., 1994), many cells were labelled by CM1 at 30 hours APF (Fig. 2C). Morphology of labelled cells was different in GMRp35 from in wild type. Instead of round, fragmented, basal cells stained in wild-type retinas, in GMRp35, the CM1-stained cells were similar to normal cells in the pigment cell lattice (Fig. 2C, and see below). Whereas in wild type the retina contained stained cells for a discrete time window around 30 hours APF, in GMRp35, CM1-stained cells were still present and morphologically normal at 48 hours APF (data not shown, but see Fig. 2D). These data show that in wildtype retina, both the morphology and disappearance of CM1labelled cells were p35 sensitive and therefore dependent on caspase function (i.e. the CM1-labelled cells were apoptotic). Based on western blotting, some or all CM1-labelling was likely to reflect cleaved Drice, whose activity was inhibited by p35. The possibility cannot be excluded that more proteins might be labelled in the tissue in addition to the Drice detected by western blotting. Tissues null mutant for drice would be required to test this possibility. Protection from cell death by p35 despite continued CM1 labelling was consistent with p35- mediated inhibition of caspase activity after zymogen cleavage, as demonstrated biochemically (Bump et al., 1995; Xue and Horvitz, 1995).

Fig. 2E-H shows high-magnification views of CM1-stained retina to illustrate cellular morphologies and subcellular staining patterns. In the wild type, most labelled cells were rounded, fragmented and basal in the epithelium (Fig. 2F). A few CM1-stained cells extended from the apical to the basal surfaces of the epithelium (Fig. 2E,F). We speculate that such cells may just be entering apoptosis. Both types of cell appeared to stain uniformly throughout their cytoplasm. By contrast, in GMRp35 retinas, CM1 staining was more concentrated around the cell periphery, and was excluded from the nucleus (Fig. 2G,H). All the labelled cells extended from the apical to the basal surfaces of the epithelium, and seemed morphologically indistinguishable from other normal cells of the pigment cell lattice.

Fig. 2I shows a pupal retina from GMRp35 double-labelled for Notch to identify all cells of the pigment cell lattice (Fehon et al., 1991). A subset of the $\mathrm{N}$-expressing cells were labelled with CM1. The labelling pattern varied from one ommatidium to the next. On average there were $6.84 \mathrm{CM} 1$-labelled cells around each ommatidium. As half were of the secondary type (shared between two ommatidia) and half tertiary (shared between three ommatidia), this corresponded to 2.9 CM1labelled cells per ommatidial unit.

\section{Caspase antibody labelling of the eye imaginal disc}

Little cell death occurs in the larval eye imaginal disc and no precise pattern has been described. Mutations such as Ellipse that prevent ommatidial differentiation result in extensive apoptosis and CM1 labelling among the unspecified cells, and were examined first as a guide (Wolff and Ready, 1991; Baker and Rubin, 1992; Kylsten and Saint, 1997; Baker and Yu, 2001). The dying cells in the Ellipse mutant were rescued by p35 expression and differentiated as pigmented cells; the number of ommatidia was not increased by preventing cell death (Fig. 2J,K). Like apoptotic cells in the pupal retina, the morphology and lifetime of CM1-labelled cells were altered by 

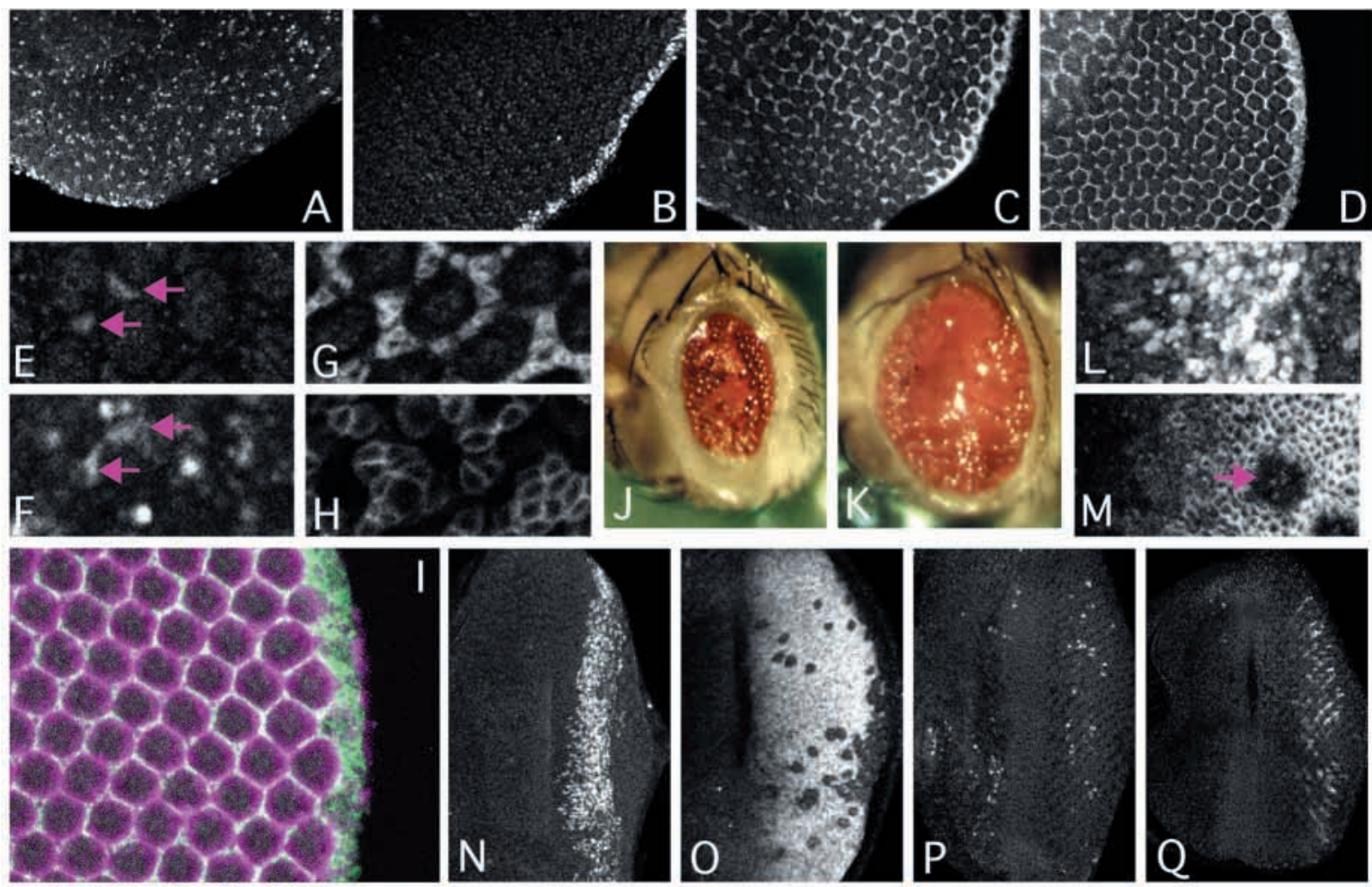

Fig. 2. (A,B) Wild-type retinas labelled with CM1 antiserum. (A) 30 hours APF. (B) 43 hours APF. (C,D) GMRp35 retinas labelled with CM1 antiserum. (C) 30 hours APF. (D) 43 hours APF. (E,F) Enlargement of wild-type retina, 30 hours APF. (E) Apical. (F) Basal. Most labelled cells are apoptotic and basal. A minority also extend apically and are more normal morphologically (arrows). (G,H) Enlargement of GMRp35 retina, 30 hours APF. (G) Apical. (H) Basal. All the labelled cells are morphologically typical of the pigment cell lattice and extend from apical to basal. Note that activated caspases are excluded from the nuclei. (I) GMRp35 retina (43 hours APF) labelled with CM1 antiserum (green) and for Notch protein (magenta). (J) Eye from Elp/Elp adult. (K) Eye from Elp/Elp; GMRp35 adult. Facet number is comparable with J, but the pigmented, unfaceted region is much larger. (L) Enlargement of Elp/Elp eye disc. Weakly labelled cells are first seen around column 5. Labelling intensifies and cells progressively round up and fragment until most labelled cells have been eliminated posterior to column 15 . (M) Enlargement of Elp/Elp; GMRp35 eye disc, apical plane. Weakly labelled cells are first seen around column 5. Labelling intensifies around the cell boundaries and highlights the normal cellular morphology. Labelled cells are maintained in the disc, not lost from the epithelium. Note that ommatidia (arrow) are protected. (N-Q) Eye imaginal discs labelled with CM1 antiserum. (N) Elp/Elp. Most cells in columns 7-15 are apoptotic. (O) Elp/Elp; GMRp35. Most undifferentiated cells posterior to the furrow are labelled. Occasional ommatidia and surrounding cells are unlabelled. In contrast to $\mathrm{N}$, labelled cells are not lost from the back of the disc. (P) Oregon R $(w)$. Scattered cell deaths are seen posterior to column 7. (Q) GMRp35. Cells protected from cell death label with CM1 but do not have apoptotic morphology.

p35. In the presence of p35 the basal, rounded, fragmented, typically apoptotic CM1-stained cells between columns 7-15 were replaced by cells resembling normal inter-ommatidial cells, labelled around the cell periphery. Such cells persisted all the way to the posterior margin of the eye disc, corresponding to at least 30 hours of continuous CM1 staining (Fig. 2L-O).

The smaller number of cells labelled by CM1 in wildtype discs had similar properties. Apoptotic cells between differentiating ommatidial clusters in columns 7-15 were replaced in GMRp35 by labelled cells resembling normal interommatidial cells and persisting to the posterior of the eye disc (Fig. 2P,Q). Fewer CM1-positive cells were seen in eye discs from the Canton $\mathrm{S}$ wild-type strain than from Oregon $\mathrm{R}$ laboratory strains (data not shown).

CM1 staining of GMRp35 was studied to infer the distribution of dying cells (Fig. 3). Staining was not even across the eye discs. Instead, foci of staining were seen against a background of minimal labelling. The position of foci varied from eye disc to eye disc (Fig. 3A-D). As survival depends on ommatidial signals to activate EGFR survival signalling (Baker and Yu, 2001), we speculate that the death-rich regions might be areas where ommatidia initiated with greater than average spacing in the morphogenetic furrow so that some interommatidial cells were too distant from survival signals. Averaging across the entire eye disc, $0.3 \mathrm{CM} 1$-stained cells were observed per ommatidial cluster in GMRp35 (Oregon R) discs (Fig. 3A,B) and 0.2 CM1-stained cells per ommatidial cluster in GMRp35 (Canton S) (Fig. 3C,D).

\section{Role of Dronc in caspase activation}

CM1-staining of p35-protected cells suggested that effector caspases such as Drice were processed in the presence of baculovirus p35 and posed the question of how this occurred. Activation might be caspase-independent or mediated by p35independent caspases. The Drosophila caspase Dronc is not inhibited by p35 (Meier et al., 2000b; Hawkins et al., 2000). Dronc has an active site sequence that is distinct from other caspases and can cleave after Glu as well as Asp.

Dominant-negative Dronc (Dronc-DN) was used to test 

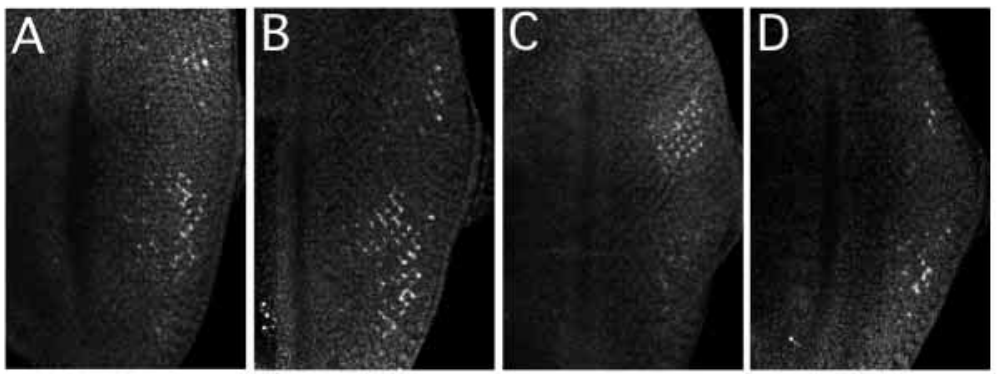

Fig. 3. Cell death in wild-type eye discs. GMRp35/+ eye discs labelled with the CM1 antiserum. Regions of moderate labelling vary in location between individuals. (A,B) Oregon R background. (C,D) Canton $\mathrm{S}$ background.

whether Dronc was required for activation of effector caspases in retina. Fig. 4 shows data for pupal retinas. Similar results were also obtained from eye discs. Neither GMR-Dronc nor GMR-Dronc-DN affected CM1-labelled cells, which had apoptotic morphology as in the wild-type background (Fig. $4 \mathrm{~A}-\mathrm{C})$. These data confirm previous results that Dronc is not essential for cell death in wild-type retinas (Meier et al., 2000b; Hawkins et al., 2000). In addition GMR-Dronc had no effect on CM1-labelling of GMR-p35 (Fig. 4D,E). By contrast, CM1labelling was not detected in eye discs and pupal retinas coexpressing GMR-Dronc-DN and GMR-p35 (Fig. 4F). These results show that p35-independent activation of effector caspases depends on Dronc.

There must be also be Dronc-independent caspase activation, because GMR-Dronc-DN did not prevent CM1 labelling in the absence of $\mathrm{p} 35$. Such activation is probably due to other caspases, because it is eliminated by p35 co-expression in GMR-Dronc-DN, GMRp35. If this was the case, CM1labelling was expected to be sensitive to DIAP1, which inhibits activation of all caspases including Dronc (Hawkins et al., 2000; Meier et al., 2000b). Consistent with this notion, CM1 labelled fewer retinal cells in GMRDIAP1 pupae (Fig. 4G). Unlike GMRp35 (Fig. 4D), DIAP1 appeared to suppress apoptosis upstream of caspase activation, consistent with
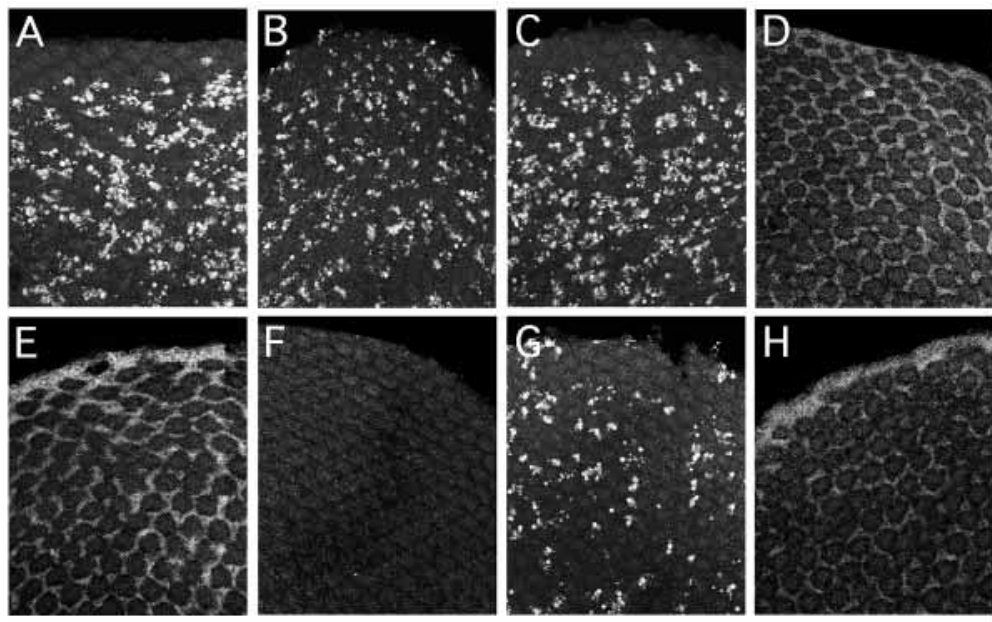

Fig. 4. Pupal retinas labelled with CM1 antiserum 30 hours APF. (A) Wild type. (B) GMRdronc/t. (C) GMRdroncDN/+. (D) GMRp35/+. (E) GMRp35/ GMRdronc. (F) GMRp35/GMRdroncDN. (G) GMRDIAP1. (H) GMRDIAP1/ GMRp35.
DIAP1-suppression of caspase zymogen cleavage. p35-sensitive morphology of the cells still labelled by CM1 in GMRDIAP1 confirmed that the cells were apoptotic (Fig. 4H). Taken together, our data indicate that effector caspases are redundantly activated by Dronc and by at least one other, p35-sensitive activator caspase. There was no evidence for caspaseindependent activation of effector caspases.

Survival of cells containing active Dronc may have developmental consequences in GMR-p35. GMR-p35 adults have a roughened eye that had been attributed to survival of supernumerary retinal cells (Hay et al., 1994) (Fig. 5A). Cell death is also reduced in GMRDIAP1 but the eyes were not roughened (Fig. 5B). Our findings suggested persistent Dronc activity as a possible cause of eye roughening in GMRp35. Alternatively, p35 expression might affect eye morphology independently of caspases. DIAP1, Dronc and Dronc-DN were expressed ectopically to distinguish these possibilities. GMR-DIAP and GM-Dronc-DN each suppressed eye roughening in GMR-p35, but GMR-Dronc did not (compare Fig. 5C,D with 5E). In the absence of GMRp35, none of GMR-DIAP, GM-Dronc, GMRDronc-DN or their combinations had any effect (Fig. 5F-I). As Dronc is the only DIAP1-sensitive caspase still active in the presence of p35 (Hawkins et al., 2000; Meier et al., 2000b) (Fig. 4G-H), each of these findings showed that Dronc activity was required for the abnormal eye morphology in GMRp35 (see Fig. 5 legend). What cellular effects of Dronc activity lead to eye roughening have not been determined.

\section{Mutations affecting cell death in the pupal retina}

Approximately 2.9 cells die for each ommatidium in the pupa around 30 hours APF. It has been suggested that hid is important for sequestration of DIAPs during cell death in the eye (Bergmann et al., 1998; Kurada and White, 1998; Wang et al., 1999; Goyal et al., 2000; Meier et al., 2000b). CM1 staining was completely absent in hid mutant pupal retinas, indicating that hid was required for apoptosis upstream of caspase activation (Fig. 6A,B). CM1 staining was also reduced by GMRDIAP1 (Fig. 4G), consistent with the model that hid acts through DIAP1 in pupal retina as in embryos (Wang et al., 1999).

Pupal apoptosis can be prevented by ectopic Ras activation. This suggested that receptor tyrosine kinases such as the EGFR might mediate survival (Miller and Cagan, 1998; Sawamoto et al., 1998). The temperature-sensitive allele egfrtsla was used to test the requirement for EGFR in survival. Reduced EGFR activity at the restrictive temperature led to a large increase in cells with activated caspases (Fig. 6C). Thus, EGFR activity was required for pupal cell survival.

Cell death in the pupal retina requires expression of $N$ (Cagan and Ready, 1989). When $N$ function was reduced in the mutations $N^{f a g}$ or $N^{l l-N t s l}$, the number of CM1 stained cells was reduced by about $80 \%$ (Fig. $6 \mathrm{D}, \mathrm{E})$. Thus, $N$ mutations blocked cell death upstream of CM1 staining, suggesting that $\mathrm{N}$ affected caspase zymogen cleavage, as did hid and EGFR, and unlike p35.

The opposing roles of EGFR and $\mathrm{N}$ signalling were 


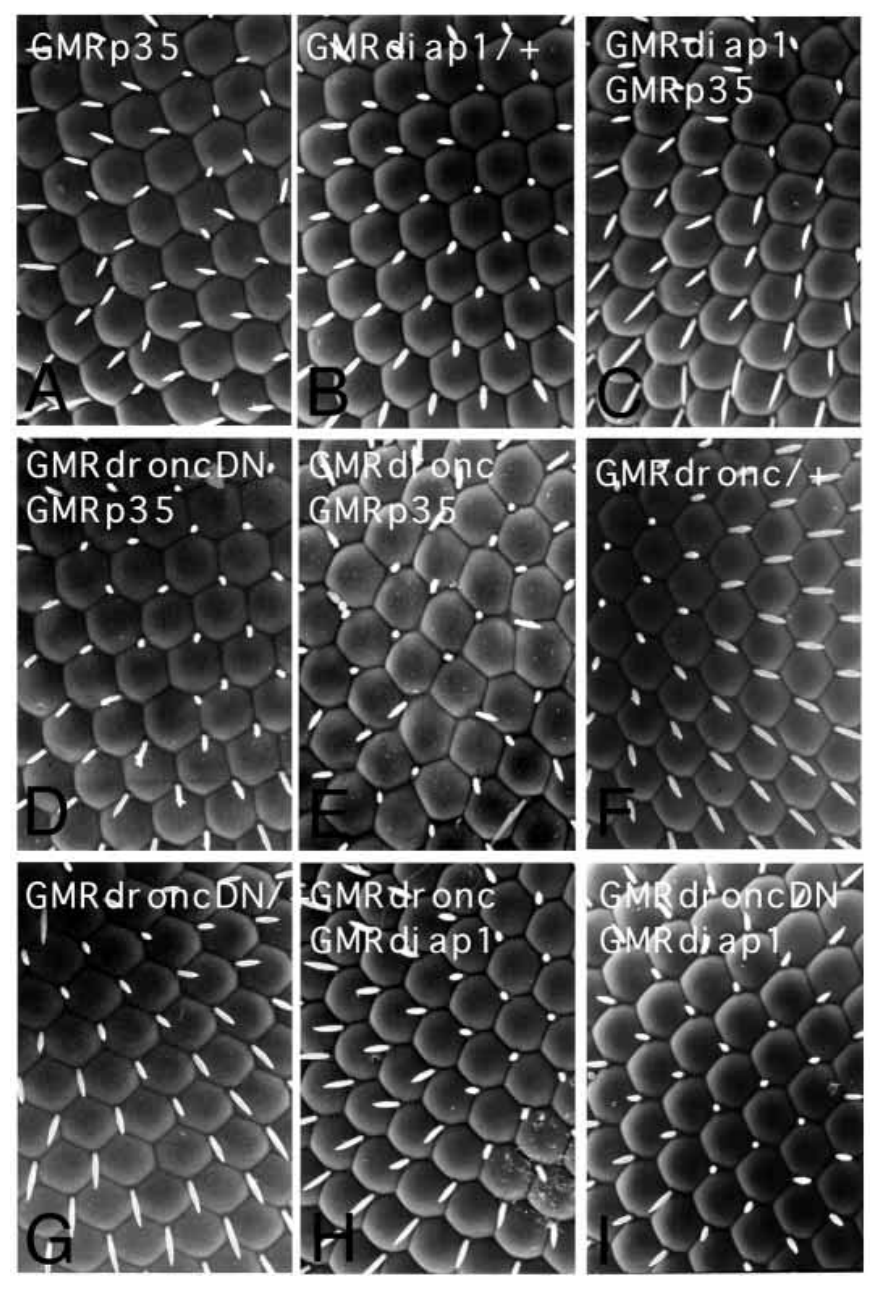

Fig. 5. Scanning electron micrographs of adult eye surfaces. (A) GMRp35. Mild eye roughening is manifested by subtle variations in facet size and shape and orientation of interommatidial bristles. (B) GMRDIAP1/+. Eye morphology was normal despite suppression of cell death (Hay et al., 1995).

(C) GMRDIAP1/GMRp35. Eye morphology was rescued compared with GMRp35. If eye roughness was caused by survival of supernumerary cells, eye roughness would be expected to be enhanced or unaffected by co-expression of DIAP1 and p35. If p35 had effects independent of cell survival, eye roughness would be expected to be unaffected by co-expression of DIAP1 and p35. If Dronc activity perturbed eye development independently of cell survival, eye roughness would be expected to be reduced by coexpression of DIAP1 and p35 because DIAP1 inhibits Dronc (Hawkins et al., 2000; Meier et al., 2000b). (D) GMRDroncDN/GMRp35. Eye morphology was rescued when compared with GMRp35, as predicted if Dronc activation were responsible for the eye roughening that occurred when $\mathrm{p} 35$ blocked cell death.

(E) GMRDronc/GMRp35. Mild eye roughening was similar to GMRp35. (F) GMRDronc/+. Eye morphology was normal.

(G) GMRDronc-DN/+. Eye morphology was normal.

(H) GMRDronc/GMRDIAP1. Eye morphology was normal.

(I) GMRDronc-DN/GMRDIAP1. Eye morphology was normal.

consistent with several models of survival control that made different predictions for epistasis. If $\mathrm{N}$ promoted cell death by antagonising EGFR survival signalling, then $N$ egfr double mutants would be expected to show levels of cell death similar to egfr mutants. Conversely, if EGFR promoted survival by antagonising apoptotic $\mathrm{N}$ signalling, then $N$ egfr double mutants would be expected to show reduced cell death, as do $N$ mutants. Finally, if $\mathrm{N}$ and EGFR acted independently to modulate the pattern of cell death, $N$ egfr double mutants would be expected to show an intermediate pattern of cell death.

Double mutant retinas were examined to distinguish the models. Caspase activation was seen in many dying cells in $N^{f a g} ;$ egfrtsla or $N^{l l-N t s l ;}$ egfrtsla mutants, similar to egfrtsla alone (Fig. 6F,G). This was consistent with a downstream role for EGFR, and indicated that $\mathrm{N}$ was required to overcome EGFR survival signalling so that cell death could occur.

\section{Mutations affecting cell death in the eye disc}

Less than one cell per ommatidium died in the eye imaginal discs of wild type flies (Fig. 3). As in pupal retina, cell survival depends on EGFR signalling (Baker and Yu, 2001). The roles of DIAP1 and hid had were examined in eye discs. Compared with wild type, CM1 staining was completely absent from eye discs from hid mutant larvae (Fig. 2H, Fig. 7A). The hid phenotype appeared semi-dominant, as the number of CM1stained cells was also reduced in hid/+ heterozygotes (Fig. 7B). By contrast, CM1 staining was unaffected by targeted DIAP1 expression, and was similar to wild type in eye discs from GMRDIAP1 larvae (Fig. 7C). To confirm that CM1-positive cells in GMRDIAP1 were indeed apoptotic, eye discs were examined from GMRDIAP1 GMRp35 larvae. The morphology of CM1-positive cells reverted to resemble normal cells when p35 was co-expressed, indicating that CM1-positive cells in GMRDIAP1 had active caspases and were apoptotic (Fig. 7D). These data confirm that hid was required for cell death in wildtype eye discs. Unlike pupal retina, however, the hid phenotype was not mimicked by overexpressing DIAP1. In a further difference from pupal retina, $N$ function was not required for CM1-labelling of eye discs (Fig. 7E).

If EGFR signalling prevented cell death by inactivation of hid expression or function, then it was predicted that EGFR should not be required for survival in the absence of hid function. Caspase activation was examined in clones of egfr mutant cells in hid mutant larvae to test this model. CM1 labelling was undetectable, confirming that EGFR promoted survival by inactivating hid $(\mathrm{Fig}$. $7 \mathrm{~F}-\mathrm{H})$. Caspase activation was also examined in clones of egfr mutant cells from GMRDIAP1 larvae. However, CM1-staining of egfr mutant cells was not prevented by DIAP1 overexpression (Fig. 7I-L). As reported previously, in egfr mutant clones from GMR-p35 larvae, cells are still labelled for caspase activation but lack apoptotic morphology and survive, consistent with Dronc activation in the absence of EGFR (Baker and Yu, 2001). Notably, such CM1 labelling was much less prominent in egfr mutant photoreceptor cells than in egfr mutant unspecified cells (Fig. 7M-P).

\section{DISCUSSION}

The CM1 antiserum, raised against mammalian caspase 3, has been used to detect dying cells in Drosophila, as well as mammalian cells (Srinivasan et al., 1998; Baker and Yu, 2001). A particularly useful feature is the detection of cells protected by baculovirus p35 expression that would otherwise be marked 

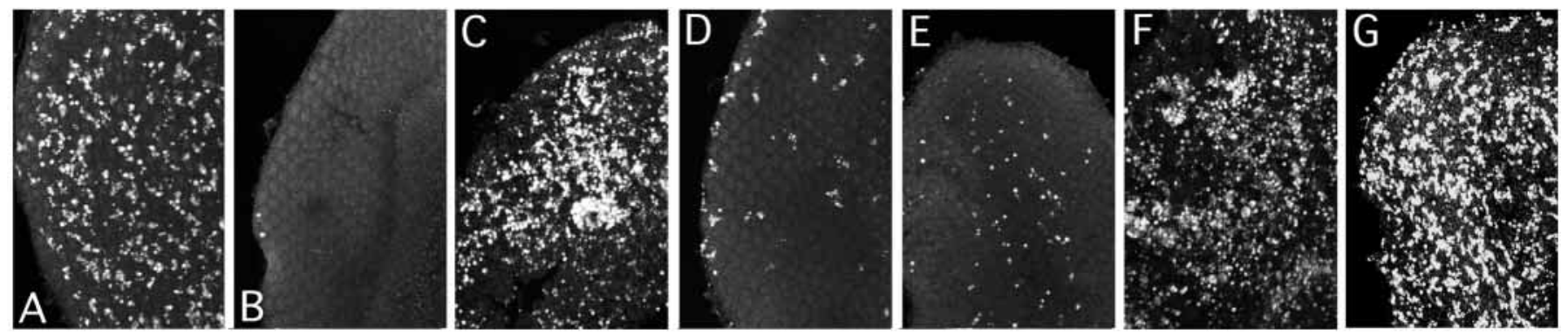

Fig. 6. Pupal retinas labelled with $\mathrm{CM} 1$ antiserum 30 hours APF $\left(25^{\circ} \mathrm{C}\right)$. (A) Wild type. (B) hid /hid. (C) egfr $r^{\text {ts } 1 a} / e g f r^{24}, 31.5^{\circ} \mathrm{C}$ for 4 hours (D) $N^{f a g}$. (E) $N^{l 1-N t s 1}, 31.5^{\circ} \mathrm{C}$ for 6 hours. (F) $N^{l 1-N t s l ;}$ egfrtsla legfr ${ }^{f 24}, 31.5^{\circ} \mathrm{C}$ for 4 hours. (G) $N^{f a g}$; egfrtsla $/$ egfr f $24,31.5^{\circ} \mathrm{C}$ for 4 hours.

for death. The morphological protection provided by p35 may permit better investigation of the location and autonomy of death and survival signals. Here, CM1 has been characterised and used to outline the pathways of apoptosis during Drosophila eye development. The results are summarised in Fig. 8.

On western blots the CM1 antibody detected activated Drice but not the Drice zymogen; Drice was also the Drosophila sequence most similar to the immunising peptide. Definitive evidence that CM1-stained cells were apoptotic comes from the dependence of embryonic CM1-staining on the 75C1,2 chromosome region, and from the p35-sensitivity of larval and pupal CM1-stained cells (Figs 1,2). The morphology of all CM1-stained cells was altered by p35 expression. In the presence of $\mathrm{p} 35, \mathrm{CM} 1$-stained cells all became
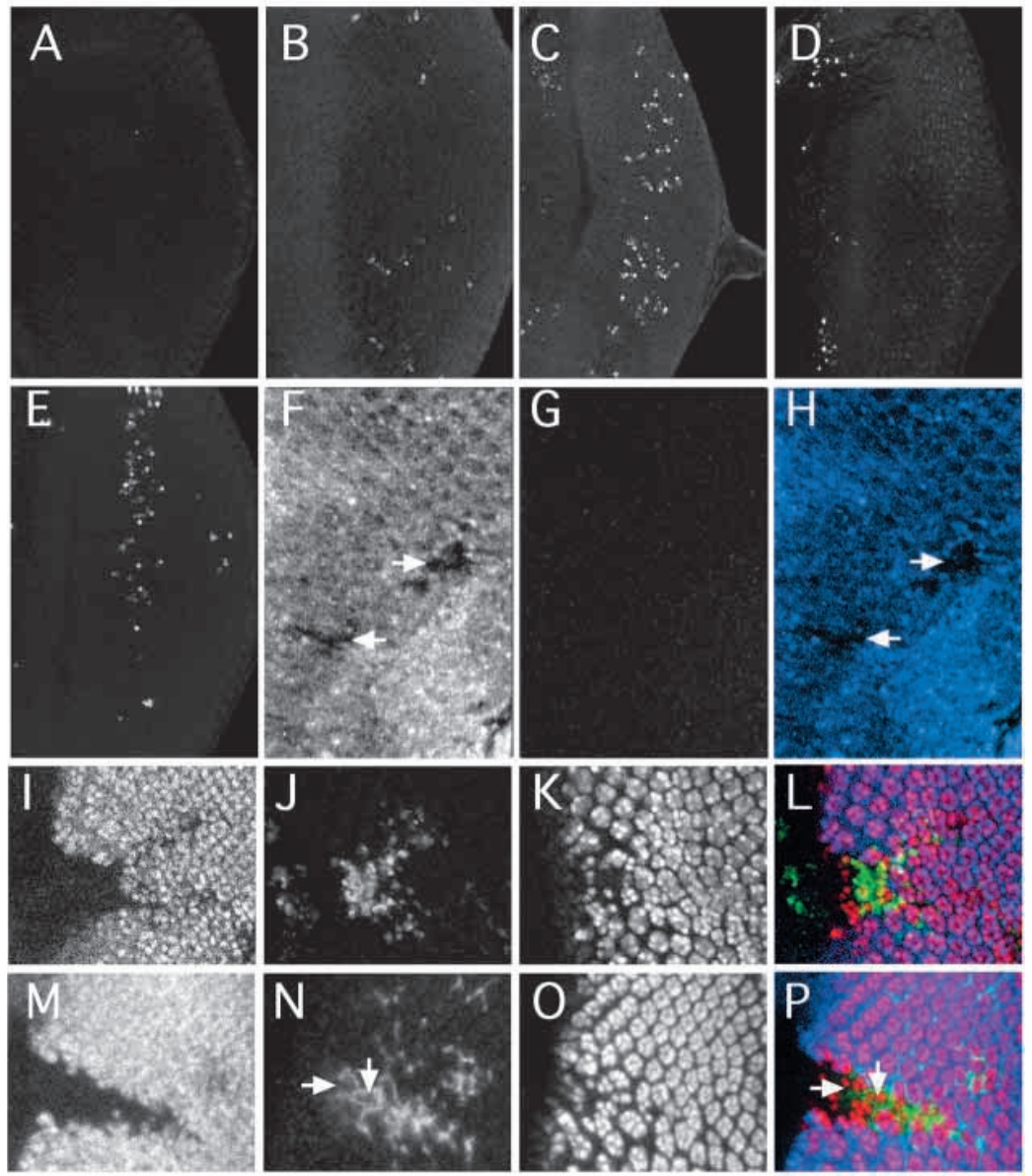

indistinguishable from normal cells by morphological criteria, and were not distinguishable except by CM1 staining. As baculovirus $\mathrm{p} 35$ blocks cell death by inhibiting caspase activity (Bump et al., 1995; Xue and Horvitz, 1995), p35-dependent morphology of CM1-stained cells showed that such morphology depended on caspase activity in the cells, which were therefore apoptotic. Our results show that only apoptotic cells were labelled by the CM1 antiserum. Apoptotic cells that were unlabelled by CM1 might also exist, although none have been noticed.

We discuss our remaining results on the simplest assumption that the CM1 staining of apoptotic cells mainly reflected processing of Drice. It should be emphasised, however, that no drice null mutations are available to test definitively whether activated Drice is the only protein recognised by the CM1 antibody in tissue samples. It cannot be excluded that, in tissue, CM1 might label other proteins as well as Drice, if such proteins were specific to apoptotic cells.

\section{Intracellular death pathway}

Our results indicate that effector caspases such as Drice can be processed in the presence of baculovirus $\mathrm{p} 35$. We found that the initiator caspase Dronc was responsible for effector caspase processing in p35 expressing cells (Fig. 4). p35insensitive caspases are also thought to initiate cell death in other insect cells (Manji and Friesen, 2001). In Drosophila eye development, Dronc always functioned redundantly with other, p35-

Fig. 7. Eye imaginal discs labelled with $\mathrm{CM} 1$ antiserum. (A) hid/hid. (B) hid/+. (C) GMRDIAP1 /GMRDIAP1. (D) GMRDIAP1/GMRp35. (E) $N^{l l-N t s l}, 31.5^{\circ} \mathrm{C}$ for 4 hours. (F-H) egfrf24 clones in hid/hid larva. (F) ArmlacZ. Arrows indicate the small clones that result when Minutes are not used. (G) CM1 labelling. (H) F,G combined, showing complete absence of apoptosis from clones (arrows). (I-L) egfr f 24 clones in

GMRDIAP1 larva. (I) ArmlacZ. (J) CM1 labelling.

(K) ELAV labelling of differentiating photoreceptors. (L) Combination of I-K showing apoptosis of egfr mutant cells. (M-P) egfr ${ }^{24}$ clones in GMRp35 larva. (M) ArmlacZ. (N) CM1 labelling. (O) ELAV labelling of differentiating photoreceptors. (P)M-O combined. Contrast morphology of CM1-labelled cells with the apoptotic cells in J. Note reduced CM1 labelling of differentiating egfr $f^{24}$ photoreceptors (arrows in N,P). 


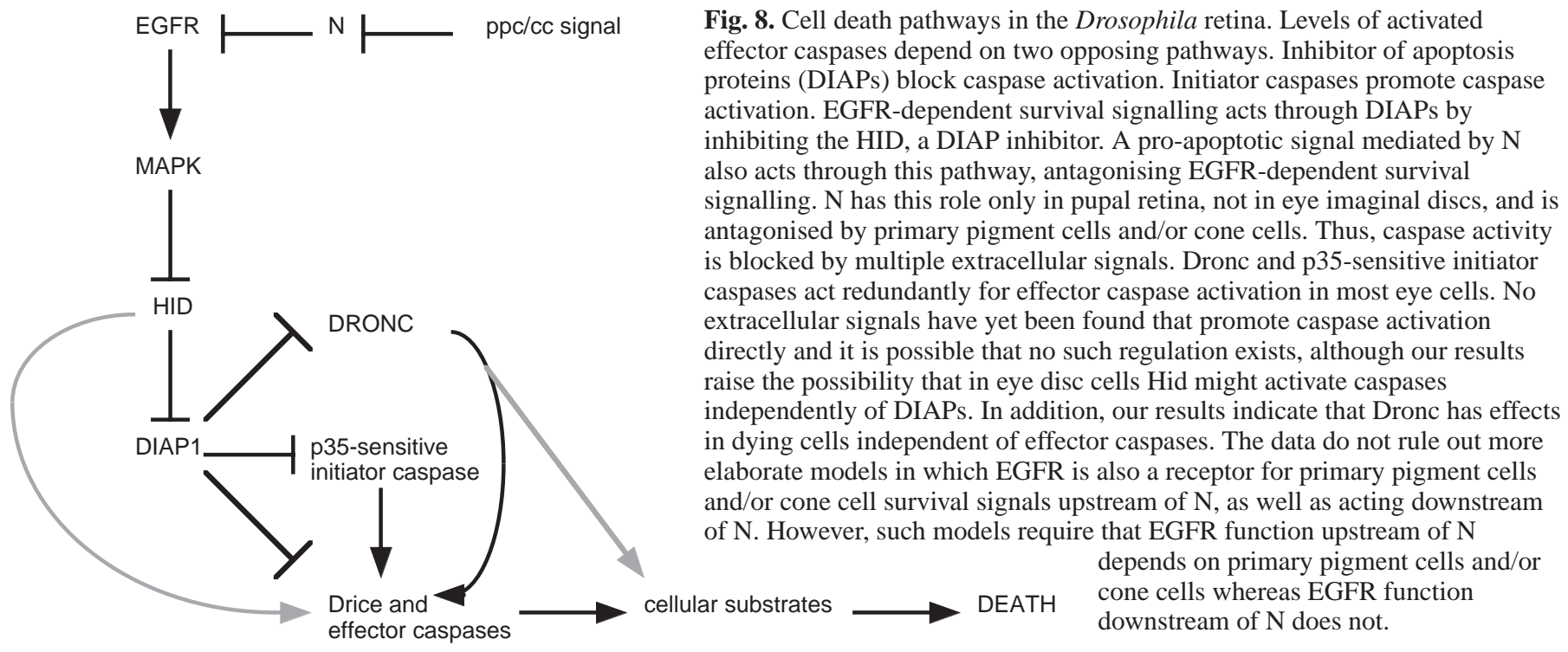

Fig. 8. Cell death pathways in the Drosophila retina. Levels of activated proteins (DIAPs) block caspase activation. Initiator caspases promote caspase activation. EGFR-dependent survival signalling acts through DIAPs by inhibiting the HID, a DIAP inhibitor. A pro-apoptotic signal mediated by $\mathrm{N}$ also acts through this pathway, antagonising EGFR-dependent survival and by primary pigment cells and/or cone cells. Thus, caspase activity extracellular signals have yet been found that promote caspase activation raise the possibility that in eye disc cells Hid might activate caspases independently of DIAPs. In addition, our results indicate that Dronc has effects in dying cells independent of effector caspases. The data do not rule out more elaborate models in which EGFR is also a receptor for primary pigment cells and/or cone cell survival signals upstream of $\mathrm{N}$, as well as acting downstream of $\mathrm{N}$. However, such models require that EGFR function upstream of $\mathrm{N}$ cone cells whereas EGFR function downstream of $\mathrm{N}$ does not.

sensitive initiator caspases. Such redundancy explains why Dronc-DN had no effect in earlier studies of eye development. Dronc has been implicated in embryonic cell death by RNA interference studies (Quinn et al., 2000).

If feedback of effector caspases on their own activation was essential for cell death, we would expect Dronc alone to be insufficient for CM1 labelling in p35-expressing cells. By contrast, CM1 labelling persisted in most cells, indicating that feedback of effector caspases was dispensable for the activation of at least one effector caspase. However, our results do not exclude the possibility that feedback makes a quantitative contribution to the pace of death or is required for a subset of effector caspases. Results were different for neuronal photoreceptor cells. R8 photoreceptor survival is rescued in the absence of EGFR by p35 expression, but such rescued R8 cells were not labelled strongly by CM1 (Kumar et al., 1998; Baker and Yu, 2001) (Fig. 7M-P). It is possible that amplification of the apoptotic cascade might be more important for apoptosis of $\mathrm{R} 8$ precursors than for unspecified cells. It is also possible that R8 cell apoptosis involved different effector caspases or was initiated independently of Dronc.

Our findings suggest that Dronc may cleave other substrates in dying cells in addition to activating p35-sensitive effector caspases. Mild eye roughening that was seen when p35 was expressed in the eye, was found to depend on Dronc activity (Fig. 5). It seemed unlikely that the rough eye could be due to some downstream effector caspases escaping the p35 inhibition, because DIAP1 overexpression blocked cell death less effectively than p35 but did not cause eye roughening. We speculate that Dronc might have cellular targets other than downstream caspases and that cleavage of such targets affected eye morphology. However, these data provide no evidence for Dronc activity, except in cells that would normally die. The simplest model is that Dronc might have another role in cell death in addition to activating effector caspases (Fig. 8). Our data do not support any effect of p35 other than its inhibition of caspases, as the eye roughening caused by p35 was suppressed by co-expression of DIAP1 or Dronc-DN.

Mutations of hid reduce cell death in pupal eye development (Kurada and White, 1998). We found hid to be absolutely required for caspase activation in both eye disc and pupal retina. Cell death was reduced even in hid/+ heterozygotes, consistent with dominant effects of hid in modifier screens (Fig. 6A,B; Fig. 7A,B). hid was required for caspase processing, which is redundantly mediated by Dronc and other initiator caspases, so, in principle, Hid was a candidate for regulating initiator caspases. However, in embryos, hid functions to sequester DIAPs (Wang et al., 1999). In pupal retinas, DIAP overexpression mimicked hid mutation, consistent with sequestration of DIAPs by Hid. In the eye imaginal disc, however, proapoptotic Hid function was not overcome by DIAP1 overexpression, as targeted DIAP1 expression neither reduced cell death in normal eye discs (Fig. 7C,D) nor protected against cell death when EGFR function was removed in egfr mutant clones (Fig. 7I-L). It seemed unlikely that endogenous Hid levels were too high for DIAP1 to be effective, because hid was haploinsufficient for eye disc cell death. Instead, these findings raise the possibility of a proapoptotic activity of Hid that is not blocked by DIAP1. This could involve inhibiting a pathway parallel to DIAP1 that also inhibits caspase activation, or promoting activation of caspase zymogens in other ways, for which there is precedent in vertebrates (Thress et al., 1998; Thress et al., 1999) (Fig. 8).

Two other proapoptotic genes rpr and grim induce eye cell death on ectopic expression. Whether rpr or grim are required for cell death in normal eye development is uncertain because point mutants are not available. The absolute requirement for hid may indicate that rpr and grim are not active during normal eye development. As hid has been shown to be required for eye death in response to ectopic $r p r$, however, it is also possible that $r p r$ and grim have activities that depend on hid function (Kurada and White, 1998).

\section{Extracellular death pathway}

Experiments using the egfrtsla allele confirmed that EGFR was required for survival of pupal retinal cells, as suggested by prior misexpression experiments (Miller and Cagan, 1998; Sawamoto et al., 1998). EGFR is also required for survival of eye imaginal disc cells (Baker and Yu, 2001). Consistent with the model that EGFR prevents cell death by inactivating hid, 
hid was absolutely required for caspase activation in egfr mutant clones (Fig. 6F-H). Similar results have been obtained using TUNEL experiments to assess EGFR-DN-induced cell death (K. White, personal communication).

Survival in pupal retina was regulated by two further extracellular signals that were not involved in eye imaginal discs. In principle, such signals might act to modulate EGFR signalling, to regulate Hid or DIAP activity in parallel to EGFR, or to activate initiator caspases. $\mathrm{N}$ was required for caspase activation in the pupal retina (Fig. 6D,E, Fig. 7E). Epistasis experiments showed that $\mathrm{N}$ was not required for pupal cell death in the absence of EGFR function, and therefore that the normal function of $\mathrm{N}$ was to inhibit the EGFR survival signalling pathway in pupae (Fig. 6F,G). Such results placed N upstream of EGFR and indicated that $\mathrm{N}$ acted ultimately through hid and the anti-apoptotic DIAP proteins that prevent caspase activation, rather than through $\mathrm{N}$-mediated caspase activation. Survival in pupal retinas also depends on signals from primary pigment cells and/or cone cells. Such signals must antagonise proapoptotic $\mathrm{N}$ activity, as $\mathrm{N}$ is epistatic to the primary pigment cell/cone cell signal (Miller and Cagan, 1998). Together with these results, our data now imply a pathway in which primary pigment cells and/or cone cells promote survival by inhibiting activation of $\mathrm{N}$, thus preventing $\mathrm{N}$ antagonism of EGFR activity in the interommatidial cells (Fig. 8).

The essential role of EGFR now seems to be downstream of $\mathrm{N}$, whereas the cone cell/primary pigment cell signal must act upstream. Downstream EGFR function raises anew the question of identity of the primary pigment cell/cone cell signal. Primary pigment cells or cone cells do not seem essential for EGFR activation, because $\mathrm{N}$ is still required for apoptosis after ablation of these cells (Miller and Cagan, 1998). Pupal photoreceptor cells express the EGFR ligand SPI and its processing/presenting factor Rhomboid, and are one possible source of EGFR activation (data not shown). One model suggests that primary pigment cells and/or cone cells are the source of an unidentified signal or mechanism that prevents $\mathrm{N}$ activation (in particular interommatidial cells) so that EGFR survival signalling can continue (Fig. 8).

\section{Survival and death signals in eye development}

According to one view, survival signals are the critical extracellular regulators of developmental cell death (Raff, 1992). By contrast, results from C. elegans and mammals indicate that cell death depends on activation of initiator caspases to trigger the apoptotic cascade (Horvitz, 1999; Aravind et al., 2001; Wang, 2001). Homologs of the activatory components exist in Drosophila (Kanuka et al., 1999; Rodriguez et al., 1999; Zhou et al., 1999; Hu and Yang, 2000).

Our studies of eye development placed three extracellular signals in a pathway acting through EGFR and hid to regulate survival, in part through IAPs (Fig. 8). The only evidence consistent with positive regulation of apoptosis was that, in eye imaginal discs, hid appeared to promote cell death through an unidentified mechanism independent of DIAPs, and, in this case, the role of EGF receptor signalling was still to promote survival by inhibiting Hid (Fig. 8).

Our findings do not rule out other pathways that activate initiator caspases during eye development, or that such activation might be required for cell death. As hid was essential for cell death, however, pathways that activate initiator caspases independently of hid cannot be sufficient for any of the cell death that normally occurs during eye development. Because loss of EGFR survival signalling was sufficient for cell death, and EGFR survival signalling was only important to inhibit Hid, our data imply that release of hid was sufficient as well as necessary for normally occurring cell death. The data do not rule out any parallel EGFR-dependent signal to suppress caspase activation independently of hid, but such a pathway cannot be sufficient for cell death in the absence of hid. Our findings suggest that positive activators of caspase processing may not be the direct targets of extracellular regulation. It will be important to investigate survival and death signals in other organs, however, including cell deaths that occur independently of reaper, grim and Hid in ovarian nurse cells and during autophagy, whose mechanisms have yet to be determined (Buczczak and Cooley, 2000; Thummel, 2001).

We thank R. Cagan, H. Steller, C. Wesley, K. White and the Bloomington Drosophila Stock Center for genetic strains, K. White for permission to cite unpublished results, and A. Koyama and B. Mollereau for comments on the manuscript. These experiments were initiated after suggestions from R. Kitsis. Supported by the NIH grants GM47892 to N. E. B. and GM57422 to B A. H. Confocal facilities provided by AIF, AECOM.

\section{REFERENCES}

Ahmad, M., Srinivasula, S. M., Wang, L., Litwack, G., FernandesAlnemri, T. and Alnemri, E. S. (1997). Spodoptera frugiperda caspase-1, a novel insect death protease that cleaves the nuclear immunophilin FKBP46, is the target of the baculovirus protein p35. J. Biol. Chem. 272, 1421-1424.

Aravind, L., Dixit, V. M. and Koonin, E. V. (2001). Apoptotic molecular machinery: vastly increased complexity I vertebrates revealed by genome comparisons. Science 291, 1279-1284.

Baker, N. E. (2001). Cell proliferation, survival, and death in the Drosophila eye. Semin. Cell Dev. Biol. 12, 499-507.

Baker, N. E. and Rubin, G. M. (1992). Ellipse mutations in the Drosophila homologue of the EGF receptor affect pattern formation, cell division, and cell death in eye imaginal discs. Dev. Biol. 150, 381-396.

Baker, N. E. and Yu, S.-Y. (2001). The EGF receptor defines domains of cell cycle progression and survival to regulate cell number in the developing Drosophila eye. Cell 104, 699-708.

Bergmann, A., Agapite, J., McCall, K. and Steller, H. (1998). The Drosophila gene hid is a direct molecular target of Ras-dependent survival signaling. Cell 95, 331-341.

Bialik, S., Cryns, V. L., Drinic, A., Miyata, S., Wollowick, A. L., Srinivasan, A. and Kitsis, R. N. (1999). The mitochondrial apoptotic pathway is activated by serum and glucose deprivation in cardiac myocytes. Circ. Res. 85, 403-414.

Buczczak, M. and Cooley, L. (2000). Eggs to die for: cell death during oogenesis. Cell Death Differ. 7, 1071-1074.

Budihardjo, I., Oliver, H., Lutter, M., Luo, X. and Wang, X. (1999). Biochemical pathways of caspase activation during apoptosis. Annu. Rev. Cell Dev. Biol. 15, 269-290.

Bump, N. J., Hackett, M., Hugunin, M., Seshagiri, S., Brady, K., Chen, P., Ferenz, C., Franklin, S., Ghayer, T., Li, P. et al. (1995). Inhibition of ICE family proteases by baculovirus antiapoptotic protein p35. Science $\mathbf{2 6 9}$, 1885-1888.

Cagan, R. L. and Ready, D. F. (1989). Notch is required for successive cell decisions in the developing Drosophila retina. Genes Dev. 3, 1099-1112.

Chinnaiyan, A. M. and Dixit, V. M. (1996). The cell-death machine. Curr. Biol. 6, 555-562.

Chong, M. J., Murray, M. R., Gosink, E. C., Russell, H. R., Srinivasan, A., Kapsetaki, M., Korsmeyer, S. J. and McKinnon, P. J. (2000). Atm and Bax cooperate in ionizing radiation-induced apoptosis in the central nervous system. Proc. Natl. Acad. Sci. USA 97, 889-894. 
Cryns, V. and Yuan, J. (1998). Proteases to die for. Genes Dev. 12, 15511570.

Ekert, P. G., Silke, J. and Vaux, D. L. (1999). Caspase inhibitors. Cell Death Differ. 6, 1081-1086.

Fehon, R. G., Johansen, K., Rebay, I. and Artavanis-Tsakonas, S. (1991). Complex cellular and subcellular regulation of Notch expression during embryonic and imaginal development of Drosophila: implications for Notch function. J. Cell Biol. 113, 657-669.

Finn, J. T., Weil, M., Archer, F., Siman, R., Srinivasan, A. and Raff, M. C. (2000). Evidence that Wallerian degeneration and localized axon degeneration induced by local neurotropin degeneration do not involve caspases. J. Neurosci. 20, 1333-1341.

Goyal, L., McCall, K., Agapite, J., Hartweig, E. and Steller, H. (2000) Induction of apoptosis by Drosophila reaper, hid and grim through inhibition of IAP function. EMBO J. 19, 589-597.

Grether, M. E., Abrams, J. M., Agapite, J., White, K. and Steller, H. (1995). The head involution defective gene of Drosophila melanogaster functions in programmed cell death. Genes Dev. 9, 1694-1708.

Gu, C., Casaccia-Bonnefil, P., Srinivasan, A. and Chao, M. V. (1999). Oligodendrocyte apoptosis mediated by caspase activation. J. Neurosci. 19 3043-3049.

Hartmann, A., Hunot, S., Michel, P. P., Muriel, M. P., Vyas, S., Faucheux, B. A., Mouatt-Prigent, A., Turmel, H., Srinivasan, A., Ruberg, M. et al. (2000). Caspase-3: a vulnerability factor and final effector in apoptotic death of dopaminergic neurons in Parkinson's disease. Proc. Natl. Acad. Sci. USA 97, 2875-2880.

Hawkins, C. J., Wang, S. L. and Hay, B. A. (1999). A cloning method to identify caspases and their regulators in yeast: identification of Drosophila IAP1 as an inhibitor of the Drosophila caspase DCP-1. Proc. Natl. Acad. Sci. USA 96, 2885-2890.

Hawkins, C. J., Yoo, S. J., Peterson, E. P., Wang, S. L., Vernooy, S. Y. and Hay, B. A. (2000). The Drosophila caspase DRONC cleaves following glutamate or aspartate and is regulated by DIAP1, HID, and GRIM. J. Biol. Chem. 275, 27084-27093.

Hay, B. A., Wolff, T. and Rubin, G. M. (1994). Expression of baculovirus P35 prevents cell death in Drosophila. Development 120, 2121-2129.

Hay, B. A., Wasserman, D. A. and Rubin, G. M. (1995). Drosophila homologs of baculovirus inhibitor of apoptosis proteins function to block cell death. Cell 83, 1253-1262.

Horvitz, H. R. (1999). Genetic control of programmed cell death in the nematode Caenorhabditis elegans. Cancer Res. 59, 1701S-1706S

Hu, S. and Yang, X. (2000). dFADD, a novel death domain-containing adapter protein for the Drosophila caspase DREDD. J. Biol. Chem. 275 , 30761-30764

Jones, B. E., Lo, C. R., Liu, H., Srinivasan, A., Streetz, K., Valentino, K. L. and Czaja, M. J. (2000). Hepatocytes sensitized to tumor necrosis factoralpha cytotoxicity undergo apoptosis through caspase-dependent and caspase-independent pathways. J. Biol. Chem. 275, 705-712.

Kanuka, H., Sawamoto, K., Inohara, N., Matsuno, K., Okano, H. and Miura, M. (1999). Control of the cell death pathway by Dapaf-1, a Drosophila Apaf-1/CED-4-related caspase activator. Mol. Cell 4, 757-769.

Krebs, J. F., Armstrong, R. C., Srinivasan, A., Aja, T., Wong, A. M., Aboy, A., Sayers, R., Pham, B., Vu, T., Hoang, K. et al. (1999). Activation of membrane-associated procaspase-3 is regulated by Bcl-2. J. Cell Biol. 144, 915-926.

Kumar, S. (1999). Mechanisms mediating caspase activation in cell death. Cell Death Differ. 6, 1060-1066.

Kurada, P. and White, K. (1998). Ras promotes cell survival in Drosophila by downregulating hid expression. Cell $\mathbf{9 5}, 319-329$

Kylsten, P. and Saint, R. (1997). Imaginal tissues of Drosophila melanogaster exhibit different modes of cell proliferation control. Dev. Biol. 192, 509-522.

Kumar, J. P., Tio, M., Hsiung, F., Akopyan, S., Gabay, L., Seger, R., Shilo, B.-Z. and Moses, K. (1998). Dissecting the roles of the Drosophila EGF receptor in eye development and MAP kinase activation. Development 125, 3875-3885.

LaCount, D. J., Hanson, S. F., Schneider, C. L. and Friesen, P. D. (2000). Caspase inhibitor p35 and inhibitor of apoptosis Op-IAP block in vivo proteolytic activation of an effector caspase at different steps. J. Biol. Chem. 275, 15657-15664.

Manji, G. A. and Friesen, P. D. (2001). Apoptosis in motion. An apical, P35insensitive caspase mediates programmed cell death in insect cells. J. Biol. Chem. 276, 16704-16710.

Meier, P., Finch, A. and Evan, G. (2000a). Apoptosis in development. Nature, 407, 796-801

Meier, P., Silke, J., Leevers, S. J. and Evan, G. I. (2000b). The Drosophila caspase DRONC is regulated by DIAP1. EMBO J 19, 598-611.

Miller, D. T. and Cagan, R. L. (1998). Local induction of patterning and programmed cell death in the developing Drosophila retina. Development 125, 2327-2335.

Nordstrom, W., Chen, P., Steller, H. and Abrams, J. M. (1996). Activation of the reaper gene defines an essential function required for both naturallyoccuring apoptosis and induced cell killing in Drosophila. Dev. Biol. 180, 227-241.

Quinn, L. M., Dorstyn, L., Mills, K., Colussi, P. A., Coombe, M., Abrams, J., Kumar, S. and Richardson, H. (2000). An essential role for the caspase Dronc in developmentally programmed cell death in Drosophila. J. Biol. Chem. 275, 40416-40424.

Raff, M. C. (1992). Social controls on cell survival and cell death. Nature 356, 397-400.

Rodriguez, A., Oliver, H., Zou, H., Chen, P., Wang, X. and Abrams, J. M. (1999). Dark is a Drosophila homologue of Apaf-1/CED-4 and functions in an evolutionarily conserved death pathway. Nat. Cell Biol. 1, 272-279.

Roy, N., Deveraux, Q. L., Takahashi, R., Salvesen, G. S. and Reed, J. C. (1997). The cIAP-1 and cIAP-2 proteins are direct inhibitors of specific caspases. EMBO J. 16, 6914-6925.

Rusconi, J. C., Hays, R. and Cagan, R. L. (2000). Programmed cell death and patterning in Drosophila. Cell Death Differ. 7, 1063-1070.

Salvesen, G. S. and Dixit, V. M. (1999). Caspase activation: the inducedproximity model. Proc. Natl. Acad. Sci. USA 96, 10964-10967.

Sawamoto, K., Taguchi, A., Hirota, Y., Yamada, C., Jin, M. H. and Okano, H. (1998). argos induces programmed cell death in the developing Drosophila eye by inhibition of the Ras pathway. Cell Death Differ. 5, 262270

Song, Z. and Steller, H. (1999). Death by design: mechanism and control of apoptosis. Trends Cell Biol. 9, M49-M52.

Srinivasan, A., Roth, K. A., Sayers, R. O., Shindler, K. S., Wong, A. M., Fritz, L. C. and Tomaselli, K. (1998). In situ immunodetection of activated caspase-3 in apoptotic neurons in the developing nervous system. Cell Death Differ. 5, 1004-1016.

Thress, K., Henzel, W., Shillinglaw, W. and Kornbluth, S. (1998). Scythe: a novel reaper-binding apoptotic regulator. EMBO J. 17, 6135-6143.

Thress, K., Evans, E. K. and Kornbluth, S. (1999). Reaper-induced dissociation of a Scythe-sequestered cytochrome $c$-releasing activity. EMBO J. 18, 5486-5493.

Thummel, C. (2001). Steroid-triggered cell death by autophagy. BioEssays 23, 677-682.

Tomlinson, A. and Ready, D. F. (1987). Neuronal differentiation in the Drosophila ommatidium. Dev. Biol. 120, 366-376.

Wang, X. (2001). The expanding role of mitochondria in apoptosis. Genes Dev. 15, 2922-2933.

Wang, S. L., Hawkins, C. J., Yoo, S. J., Muller, H. A. and Hay, B. A. (1999). The Drosophila caspase inhibitor DIAP1 is essential for cell survival and is negatively regulated by HID. Cell 98, 453-463.

White, K., Grether, G. E., Abrams, J. M., Young, L., Farrell, K. and Steller, H. (1994). Genetic control of programmed cell death in Drosophila. Science 264, 677-683.

Wolff, T. and Ready, D. F. (1991). Cell death in normal and rough eye mutants of Drosophila. Development 113, 825-839.

Xue, D. and Horvitz, H. R. (1995). Inhibition of the Caenorhabditis elegans cell-death protease CED-3 by a CED-3 cleavage site in baculovirus p35 protein. Nature 377, 248-251.

Zhou, L., Song, Z., Tittel, J. and Steller, H. (1999). HAC-1, a Drosophila homolog of APAF-1 and CED-4 functions in developmental and radiationinduced apoptosis. Mol. Cell 4, 745-755. 